\title{
Adult-onset Still's disease presenting as fever of unknown origin: a single-center retrospective observational study from China
}

\author{
Mengying Zhang", Yaman Wang", Jie Li", Jun Zhou^ \\ Department of Laboratory Medicine, the First Affiliated Hospital of Nanjing Medical University, Nanjing, China \\ Contributions: (I) Conception and design: J Zhou, M Zhang; (II) Administrative support: J Zhou, M Zhang; (III) Provision of study materials or \\ patients: Y Wang, J Li; (IV) Collection and assembly of data: All authors; (V) Data analysis and interpretation: J Zhou, M Zhang; (VI) Manuscript \\ writing: All authors; (VII) Final approval of manuscript: All authors. \\ \#These authors contributed equally to this work. \\ Correspondence to: Jun Zhou. Department of Laboratory Medicine, the First Affiliated Hospital of Nanjing Medical University, Nanjing, China. \\ Email: zhoujun5958@163.com.
}

Background: The aim of this study is to establish a clinical diagnosis model as a new evaluation indicator
for the differentiation of adult-onset Still's disease (AOSD) and other fever of unknown origin disease (FUO).
Methods: This is an observational case-control study between January 2010 and December 2018.
Laboratory parameters of AOSD group (N=91), FUO group ( $\mathrm{N}=89$ ) and control group (N=81) including
procalcitonin, C-reactive protein (CRP), ferritin, leukocyte, lymphocyte, neutrophil, lymphocyte proportion,
neutrophil proportion, red blood cell distribution width (RDW), platelet and platelet parameters were
collected. Descriptive statistics and logistic regression were performed to establish a model based on these
laboratory variables.

Results: After univariate screening, the variables including CRP, leukocyte, neutrophil, lymphocyte proportion, neutrophil proportion, ferritin and mean platelet volume (MPV) showed significant difference between AOSD and FUO groups, then a stepwise regression analysis was performed to establish a model based on these screened variables, at last ferritin, neutrophil and MPV were significantly different in the model. The results suggested that the higher value of ferritin and neutrophil, the lower value of MPV in the model indicated the higher risk to diagnose AOSD. Area under the curve (AUC) of the model was 0.909 (95\% CI: 0.855-0.947), which showed high differential diagnostic value (sensitivity: $86.6 \%$, specificity: $82.0 \%$ ).

Conclusions: The diagnosis model of AOSD and other FUO was established, with an outstanding performance for differential diagnosis.

Keywords: Adult-onset Still's disease (AOSD); fever of unknown origin disease (FUO); ferritin; diagnosis; model

Submitted Jan 20, 2020. Accepted for publication Aug 02, 2020.

doi: 10.21037/apm-20-268

View this article at: http://dx.doi.org/10.21037/apm-20-268

\section{Introduction}

Adult-onset Still's disease (AOSD) is a rare multisystem auto-inflammatory disorder, characterized with highspiking fever, arthritis/arthralgia, rash, leukocytosis with neutrophilia, elevated ferritin levels (1). Researchers have made huge efforts to reveal that infection may play a role in the disorder, however the exact etiopathogenesis is still unknown (2-6). The Yamaguchi criteria are widely used in diagnosing AOSD and experimental study (7). However, it is still a clinical challenge to make a diagnosis of AOSD as it is an exclusive diagnosis $(8,9)$.

Fever of unknown origin (FUO) are multi-causes, difficult to diagnose and time-consuming clinical diseases

\footnotetext{
^ ORCID: 0000-0002-0716-3967.
} 
defined as fever $\left(>38.3^{\circ} \mathrm{C}\right)$ lasting more than 3 weeks and remaining undiagnosed after standardized history-taking, physical examination, and certain obligatory investigations (1,10-14). Patients with hematological disease, receiving chemotherapy, glucocorticoids and other autoimmune diseases were excluded. Pizzo et al. reported that the duration of hospitalization for FUO can lengthen 19 days (15). About 15-20\% of patients evaluated for FUO had the diagnosis of AOSD (16). In clinical practice, malignancyassociated FUO, infection-associated FUO and noninfection associated FUO are most common (14). Such a heterogeneous composite often requires a thorough diagnostic work-up. There is still no effective differential diagnosis method. Therefore, for patients with FUO, we hypothesize a simple clinical diagnostic model to help clinicians to differentiate patients with AOSD from other FUO causes, to effectively save existing medical resources, relieve pain and reduce the economic burden on patients.

We present the following article in accordance with the STROBE reporting checklist (available at http://dx.doi. org/10.21037/apm-20-268).

\section{Methods}

\section{Study design and patient selection}

This is an observational case-control study. Patients ( $\geq 16$ years) initially diagnosed with AOSD and met Yamaguchi criteria (7) between January 2010 and December 2018 in our hospital were recruited (AOSD Group). Patients admitted to the same center diagnosed with other FUO and met the standard criteria $(1,14)$ [patients ( $\geq 16$ years) who had a body temperature $>38.3^{\circ} \mathrm{C}$ for at least 3 weeks without an established cause of fever after 1 week of hospitalization] during the same time were also recruited (FUO group). Patients with hematological disease, receiving chemotherapy, glucocorticoids, other autoimmune diseases and incomplete information and data were excluded. We also reviewed 81 healthy controls, who visited our hospital for medical check-ups during the same time.

Blood samples were taken when admission and measured within 2 hours [blood routine indexes were analyzed using a Sysmex XE 2100 analyzers (Sysmex, Japan), ferritin analysis was performed on Unicel DXI 800 (Beckman Coulter, America)], data of three groups were collected respectively. This study was approved by the Ethics Committee of The First Affiliated Hospital of Nanjing Medical University (Nanjing, China) (2019-SR-072) and in accordance with the
Declaration of Helsinki (as revised in 2013). We obtained patients' written consents prior to data collection.

\section{Assessment of the patients}

Admission data (initial diagnosis and before the initiation of any treatment) (results of the first blood draw) such as clinical parameters, including age, gender, fever or not, typical skin rash, arthritis/arthralgia, sore throat, myalgia, lymphadenopathy, hepatomegaly, splenomegaly and laboratory parameters, including procalcitonin, C-reactive protein (CRP), ferritin, leukocyte, lymphocyte, neutrophil, lymphocyte proportions, neutrophil proportions, red blood cell distribution width (RDW), platelet, platelet parameters of all patients were obtained and recorded. There were insufficient data to test the strength of glycosylated ferritin and IL-18 in the FUO and AOSD groups.

\section{Statistical analysis}

All statistical analysis was performed on the Statistical Package for the Social Sciences software (version 21; IBM Corporation, Armonk, NY, USA).

Kolmogorov-Smirnov analysis was used to test the distribution form of all variables. The data of descriptive analysis were expressed as either mean \pm standard deviation (SD) (normally distributed) or the median, interquartile range (non-normally distributed). The chi-square test or Fisher's exact test were used to compare categorical variables where appropriate. The non-normally and normally distributed continuous data between two groups were compared with Mann-Whitney $\mathrm{U}$ test and Student $t$ test respectively. For the multivariate analysis, the variables identified with univariate analyses were further entered into the logistic regression analysis to determine independent predictors of patient outcome. After the clinical feasibility assessment of possible parameters derived from multivariate analysis, we developed a model. $\mathrm{P}<0.05$ was used to infer statistical significance.

\section{Results}

\section{Demographic, clinical, and laboratory features of the study subjects}

The clinical characteristics of AOSD group and FUO group are shown in Table 1, Fever was found in almost all patients in both groups (98.9\% vs. 100\%). Arthralgia/arthritis could 
Table 1 Characteristics of the study cohorts

\begin{tabular}{lccr}
\hline Variables & AOSD group (N=91) (\%) & FUO group (N=89) (\%) & P value \\
\hline Fever & $90(98.9)$ & $89(100.0)$ & 0.361 \\
Arthralgia/arthritis & $60(65.9)$ & $25(28.1)$ & $<0.001$ \\
Typical skin rash & $39(42.9)$ & $13(14.6)$ & $<0.001$ \\
Sore throat & $41(45.1)$ & $11(12.4)$ & $30(33.7)$ \\
Lymphadenopathy & $26(28.6)$ & $5(5.6)$ & 0.003 \\
Myalgia & $29(31.9)$ & $6(6.7)$ & $<.140$ \\
Hepatomegaly & $10(11.0)$ & $9(11.1)$ & 0.001 \\
Splenomegaly & $12(13.2)$ & 0.7965 \\
\hline
\end{tabular}

AOSD, adult-onset Still's disease; FUO, fever of unknown origin.

be seen in 60 of 91 patients $(65.9 \%)$ and 25 of 89 patients (28.1\%) in the AOSD and FUO groups, respectively. Typical skin rash was present in 39 of 91 patients (42.9\%) and 13 of 89 patients $(14.6 \%)$ in AOSD group and FUO groups, respectively. There was no statistically significant difference in lymphadenopathy between the two groups. In addition to above, sore throat, myalgia, hepatomegaly and splenomegaly could occur in both groups.

The demographic and laboratory parameters were also compared among the three groups showing in Table 2. Thirty-two (35.2\%) patients were male in AOSD group, 33 (37.1\%) patients were male in FUO group and 41 (50.6\%) were male in control group. Median ages of patients were similar [32 (range, 16-74) years for AOSD group, 36 (range, 16-73) for FUO group and 36 (range, 22-76) for control group]. There were no significant differences in age and gender $(\mathrm{P}=0.866, \mathrm{P}=0.085)$. $\mathrm{CRP}$ and ferritin differed significantly between AOSD and FUO groups. Leukocyte, lymphocyte, neutrophil, lymphocyte proportions, neutrophil proportions, plateletcrit (PCT), mean platelet volume (MPV) and platelet distribution width (PDW) were significantly different among the three groups. However, there were also no significant differences in procalcitonin, RDW, platelet among the three groups (Table 2).

Detailed information of ferritin, neutrophil and MPV levels in patients are show in Table 2. The level of ferritin was higher in AOSD group than that in FUO group. The level of neutrophil was higher in AOSD group compared with FUO group and control group (AOSD group vs. FUO group, $\mathrm{P}<0.001$; AOSD group vs. control group, $\mathrm{P}<0.001$; FUO group vs. control group, $\mathrm{P}<0.001)$. However, MPV was lower in AOSD group compared with FUO group and higher in AOSD group compared with control group (AOSD group vs. FUO group, $\mathrm{P}<0.001$; AOSD group $v$ s. control group, P 0.001; FUO group vs. control group, $\mathrm{P}<0.001)$. The FUO group was also divided into three subgroups: Infection-associated disease $(n=38,42.7 \%)$, malignancy-associated disease $(\mathrm{n}=29,32.6 \%)$ and connective tissue-associated disease $(n=22,24.7 \%)$. Laboratory features of each subgroup are given in Table $S 1$.

\section{Establishment of clinical diagnosis regression model}

After the identification of possible factors for predicting AOSD by univariate analysis, we performed a binary logistic regression analysis to determine independent predictors of AOSD. Details of univariate and multivariate analyses are given in Table 3. After univariate screening, variables which were executed in the model including CRP, ferritin, leukocyte, neutrophil, neutrophil proportions, lymphocyte proportions and MPV, in which the variables ferritin, neutrophil and MPV were significantly different $(\mathrm{P}<0.001, \mathrm{P}<0.001, \mathrm{P}=0.016$; respectively). The higher ferritin, neutrophil and the lower value of MPV in the model indicated the higher probability to diagnose AOSD. Table 3 also showed the regression coefficients and risk score for each variable. The equation for the model is as follows: Logit $\mathrm{P}=2.304 \times$ ferritin $+2.696 \times$ neutrophil $-1.260 \times$ MPV - 5.108.

\section{Area under the curve and diagnostic cutoff value}

It is well known that the area under the curve ranged from 0.5 to 1.0 . When the AUC $<0.7$ indicated that the 
Table 2 Demographic and laboratory parameters of patients for study cohorts

\begin{tabular}{|c|c|c|c|c|}
\hline Variables & AOSD group $(\mathrm{N}=91)$ & FUO group ( $\mathrm{N}=89)$ & Controls $(\mathrm{N}=81)$ & $P$ value \\
\hline Sex, male/female & $32 / 59$ & $33 / 56$ & $41 / 40$ & 0.085 \\
\hline Procalcitonin ( $\mu \mathrm{g} / \mathrm{L})$ & $0.47 \pm 0.75^{\mathrm{a}}$ & $1.66 \pm 6.82$ & - & 0.198 \\
\hline CRP (mg/L) & $102.99 \pm 69.17^{a}$ & $69.60 \pm 66.23$ & - & 0.002 \\
\hline Leukocyte (×109/L) & $15.35 \pm 8.04^{a}$ & $7.29 \pm 4.95$ & $6.09 \pm 1.68^{\mathrm{c}}$ & $<0.001$ \\
\hline Lymphocyte (×109/L) & $1.34 \pm 0.62$ & $1.44 \pm 1.89$ & $1.87 \pm 0.54^{\mathrm{c}}$ & 0.010 \\
\hline Neutrophil (×109\%) & $13.27 \pm 8.02^{\mathrm{a}}$ & $5.12 \pm 3.79^{b}$ & $3.68 \pm 1.37^{\mathrm{c}}$ & $<0.001$ \\
\hline Lymphocyte proportions (\%) & $11.34 \pm 8.65$ & $22.78 \pm 18.73^{b}$ & $31.70 \pm 7.45^{\mathrm{c}}$ & $<0.001$ \\
\hline Platelet $\left(\times 10^{9} / \mathrm{L}\right)$ & $251.77 \pm 109.05$ & $222.67 \pm 164.41$ & $226.59 \pm 45.55$ & 0.206 \\
\hline РCT (\%) & $0.25 \pm 0.10$ & $0.23 \pm 0.15$ & $0.20 \pm 0.04^{c}$ & 0.009 \\
\hline MPV (fL) & $9.80 \pm 1.23^{\mathrm{a}}$ & $10.42 \pm 1.03^{b}$ & $8.84 \pm 1.11^{\mathrm{c}}$ & $<0.001$ \\
\hline PDW (\%) & $12.26 \pm 2.46$ & $12.15 \pm 2.24^{b}$ & $16.93 \pm 0.54^{c}$ & $<0.001$ \\
\hline
\end{tabular}

${ }^{a}$, indicates a significant difference $(P<0.05)$ between AOSD group and FUO group; ${ }^{b}$, indicates a significant difference $(P<0.05)$ between FUO group and control group; ${ }^{c}$, indicates a significant difference $(P<0.05)$ between AOSD group and control group. AOSD, adult-onset Still's disease; FUO, fever of unknown origin; CRP, C-reactive protein; RDW, red blood cell distribution width; MPV, mean platelet volume; PCT, plateletcrit; PDW, platelet distribution width.

Table 3 Univariate and multivariate analyses of factors for differentiate AOSD from FUO

\begin{tabular}{|c|c|c|c|c|c|c|c|c|c|}
\hline Variables & \multicolumn{3}{|c|}{ Univariate analysis } & \multicolumn{6}{|c|}{ Multivariate analysis } \\
\hline CRP & 2.258 & $1.22-4.17$ & 0.010 & - & - & - & - & - & - \\
\hline Ferritin & 12.75 & $6.16-26.40$ & $<0.001$ & 2.304 & 0.481 & 22.337 & 10.01 & $4.04-24.82$ & $<0.001$ \\
\hline Leukocyte & 17.17 & $8.20-35.93$ & $<0.001$ & - & - & - & - & - & - \\
\hline Lymphocyte proportions (\%) & 0.14 & $0.073-0.27$ & $<0.001$ & - & - & - & - & - & - \\
\hline Neutrophil proportions (\%) & 10.82 & $5.42-21.60$ & $<0.001$ & - & - & - & - & - & - \\
\hline MPV & 0.42 & $0.23-0.77$ & 0.007 & -1.260 & 0.485 & 6.417 & 0.28 & $0.08-0.97$ & 0.045 \\
\hline AUC & - & - & - & 0.909 & - & - & - & - & - \\
\hline
\end{tabular}

AOSD, adult-onset Still's disease; AUC, area under the curve; CRP, C-reactive protein; FUO, fever of unknown origin; MPV, mean platelet volume; PE, parameter estimate; SE, standard error.

diagnostic accuracy was low; AUC ranging between 0.7 and 0.9 has a high accuracy; and AUC $>0.9$ indicated that the diagnostic accuracy was greatly enhanced. As presented in Figure 1, AUC of the model was 0.909 (95\% CI: $0.855-$
0.947), suggesting that the model had a good differential diagnostic efficiency. Youden's index (sensitivity + specificity -1) was used to determine the best value for sensitivity and specificity (Table 4). In the model, when the cutoff point 


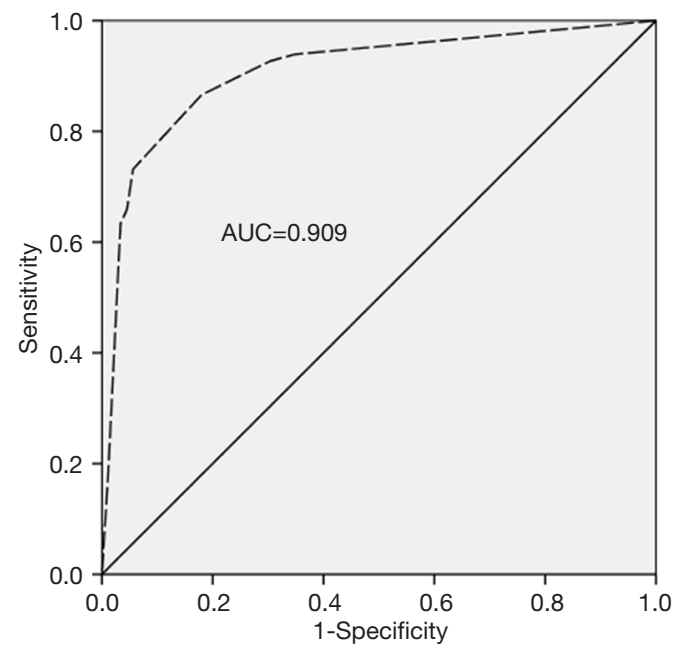

Figure 1 Characteristic feature of the curve for model $(\mathrm{P}<0.001)$.

Table 4 Cutoff point, sensitivity and specificity of the model at each value

\begin{tabular}{lccc}
\hline Cutoff point & Sensitivity (\%) & Specificity (\%) & Youden's index \\
\hline 0.135 & 93.9 & 65.2 & 0.591 \\
0.311 & 92.7 & 69.7 & 0.624 \\
0.468 & 86.6 & 82.0 & 0.686 \\
0.617 & 73.2 & 94.4 & 0.676 \\
0.754 & 65.9 & 95.5 & 0.614 \\
0.852 & 63.4 & 96.6 & 0.600 \\
0.944 & 18.3 & 98.9 & 0.172 \\
\hline
\end{tabular}

The maximum value Youden's index is the optimal value;

Youden's index $=$ sensitivity + specificity -1 .

is higher than 0.468 , the sensitivity and specificity for diagnosis of AOSD were $86.6 \%$ and $82.0 \%$, respectively. In contrast, when the cutoff point was $<0.468$, other FUO was diagnosed.

\section{Discussion}

In this study, we quantified the contribution of laboratory features rather than clinical features to detect patients who have the high likelihood of being diagnosed with AOSD as clinical features are either being ignored or with low specificity. After screening, ferritin, neutrophil and MPV were selected into the model. The larger value of ferritin and neutrophil, the smaller value of MPV in the model indicated the higher potential of AOSD.

Fever is the major clinical manifestation of AOSD and other FUO (14,17). Several epidemiological studies revealed that the AOSD was one of the most common rheumatologic-associated causes of FUO $(18,19)$. Evaluation of patients with FUO is exhausting, time-consuming, and expensive (20). At present, clinicians distinguish them mainly based on clinical characteristics, laboratory features and personal experience. Generally, ferritin, glycosylated ferritin, IL-18, CRP, Leukocyte, neutrophil, lymphocyte and RDW are mainly used to diagnose AOSD (21). However, these markers can also increase in other FUO patients, and there is no clearly defined range and clinicians can only judge based on clinical experience. In addition, the measurement of glycosylated ferritin and IL-18 is not clinically feasible as they are not available for many centers despite of their accuracy. It is still difficult to make differential diagnosis of AOSD and other FUO. Without properly and effectively guided clinical treatment, patients might accept the wrong treatment plan or delayed treatment, leading to serious adverse consequences. Therefore, making differentiation of these two clinical entities with a clinical diagnosis model is invaluable.

We first compared laboratory markers of patients with AOSD and patients with other FUO to find marker(s) which can detect patients with AOSD and patients with other FUO who have the high likelihood of being diagnosed with AOSD. The results showed that leukocyte, CRP, ferritin, neutrophil and neutrophil proportions were higher in AOSD, while Lymphocyte proportions and MPV were higher in other FUO patients. Furthermore, multivariate analysis showed ferritin, neutrophil and MPV were independent factors, so we put all of them into the model to build a model.

Ferritin is correlated with severe hyperinflammation in ICU-patients (22). To newborns with hypoxic-ischemic brain injury, neutrophil could be used as an inflammationsensitive marker (23). MPV is measured with the automatic analyzer, standing for the average platelet size. An escalated MPV is serving as the destruction and consumption of platelets (24). In this study, compared with FUO group, increased number of platelets and lower PDW were revealed in the AOSD group, this would indicate a relationship between AOSD and platelet activation.

The model has a high diagnostic value for the identification of AOSD and other FUO with high sensitivity (86.6\%), specificity (82.0\%) and AUC [0.909 (95\% CI: 0.855-0.947)], helping clinicians make diagnosis based on 
readily available clinical data, when the patient's etiological analysis is not clear or clinical features are not obvious. We recommended 0.468 as the cutoff value for diagnosing AOSD and other FUO. If the AUC is greater than cutoff point, AOSD should be diagnosed. Conversely, if the AUC is lower than the cutoff point, other FUO should be diagnosed. The model can be used widely as the included markers can be performed even in most of local hospitals.

However, there are also some shortcomings in our model. For example, due to the limited sample size, we have not yet verified the results for large sample sizes. Future studies will further optimize and verify the model. Another limitation of our study is the fact that we could not include all the potential biological markers that can differentiate AOSD from other FUO because of methodological and financial reasons (25).

In conclusion, in the present study, clinical indexes including ferritin, neutrophil and MPV were used to establish a clinical diagnostic model, which can be quantitative assistance for the diagnosis of AOSD and other FUO. Therefore, it helps clinicians differentiate AOSD and other FUO, making it easier and timelier to provide patients with optimal treatments.

\section{Acknowledgments}

Funding: None.

\section{Footnote}

Reporting Checklist: The authors have completed the STROBE reporting checklist. Available at http://dx.doi. org/10.21037/apm-20-268

Data Sharing Statement: Available at http://dx.doi. org/10.21037/apm-20-268

Conflicts of Interest: All authors have completed the ICMJE uniform disclosure form (available at http://dx.doi. org/10.21037/apm-20-268). The authors have no conflicts of interest to declare.

Ethical Statement: The authors are accountable for all aspects of the work in ensuring that questions related to the accuracy or integrity of any part of the work are appropriately investigated and resolved. This study was approved by the Ethics Committee of The First Affiliated Hospital of Nanjing Medical University (Nanjing, China)
(2019-SR-072) and in accordance with the Declaration of Helsinki (as revised in 2013). We obtained patients' written consents prior to data collection.

Open Access Statement: This is an Open Access article distributed in accordance with the Creative Commons Attribution-NonCommercial-NoDerivs 4.0 International License (CC BY-NC-ND 4.0), which permits the noncommercial replication and distribution of the article with the strict proviso that no changes or edits are made and the original work is properly cited (including links to both the formal publication through the relevant DOI and the license). See: https://creativecommons.org/licenses/by-nc-nd/4.0/.

\section{References}

1. Petersdorf RG, Beeson PB. Fever of unexplained origin: report on 100 cases. Medicine (Baltimore) 1961;40:1-30.

2. Huang SH, DeCoteau WE. Adult-onset Still's disease: an unusual presentation of rubella infection. Can Med Assoc J 1980;122:1275-6.

3. Wouters JM, van der Veen J, van de Putte LB, et al. Adult onset Still's disease and viral infections. Ann Rheum Dis 1988;47:764-7.

4. Ohta A, Yamaguchi M, Tsunematsu T, et al. Adult Still's disease: a multicenter survey of Japanese patients. J Rheumatol 1990;17:1058-63.

5. Pouchot J, Ouakil H, Debin ML, et al. Adult Still's disease associated with acute human parvovirus B19 infection.

Lancet 1993;341:1280-1.

6. Efthimiou P, Paik PK, Bielory L. Diagnosis and management of adult onset Still's disease. Ann Rheum Dis 2006;65:564-72.

7. Yamaguchi M, Ohta A, Tsunematsu T, et al. Preliminary criteria for classification of adult Still's disease. J Rheumatol 1992;19:424-30.

8. Ahn MH, Kim HA. Biomarkers for adult-onset still's disease: up-to-date. Expert Rev Mol Diagn 2019;19:655-7.

9. Jung JY, Suh CH, Kim HA. The role of damage-associated molecular pattern for pathogenesis and biomarkers in adult-onset Still's disease. Expert Rev Mol Diagn 2019;19:459-68.

10. Petersdorf RG. Fever of unknown origin. An old friend revisited. Arch Intern Med 1992;152:21-2.

11. Bleeker-Rovers CP, Vos FJ, de Kleijn EM, et al. A prospective multicenter study on fever of unknown origin: the yield of a structured diagnostic protocol. Medicine 
(Baltimore) 2007;86:26-38.

12. Bleeker-Rovers CP, Vos FJ, Mudde AH, et al. A prospective multi-centre study of the value of FDG-PET as part of a structured diagnostic protocol in patients with fever of unknown origin. Eur J Nucl Med Mol Imaging 2007;34:694-703.

13. Baymakova M, Popov GT, Andonova R, et al. Fever of unknown origin and Q-fever: a case series in a Bulgarian hospital. Caspian J Intern Med 2019;10:102-6.

14. Durack DT, Street AC. Fever of unknown origin-reexamined and redefined. Curr Clin Top Infect Dis 1991;11:35-51.

15. Pizzo PA, Lovejoy FH Jr, Smith DH. Prolonged fever in children: review of 100 cases. Pediatrics 1975;55:468-73.

16. Kucukardali Y, Oncul O, Cavuslu S, et al. The spectrum of diseases causing fever of unknown origin in Turkey: a multicenter study. Int J Infect Dis 2008;12:71-9.

17. Crispín JC, Martinez-Banos D, Alcocer-Varela J. Adultonset Still disease as the cause of fever of unknown origin. Medicine (Baltimore) 2005;84:331-7.

18. Bilgin E, Hayran M, Erden A, et al. Proposal for a simple algorithm to differentiate adult-onset Still's disease with other fever of unknown origin causes: a longitudinal prospective study. Clin Rheumatol 2019;38:1699-706.

Cite this article as: Zhang M, Wang Y, Li J, Zhou J. Adultonset Still's disease presenting as fever of unknown origin: a single-center retrospective observational study from China. Ann Palliat Med 2020;9(5):2786-2792. doi: 10.21037/apm-20-268
19. Sipahi OR, Senol S, Arsu G, et al. Pooled analysis of 857 published adult fever of unknown origin cases in Turkey between 1990-2006. Med Sci Monit 2007;13:CR318-22.

20. Naito T, Mizooka M, Mitsumoto F, et al. Diagnostic workup for fever of unknown origin: a multicenter collaborative retrospective study. BMJ Open 2013;3:e003971.

21. Feist E, Mitrovic S, Fautrel B. Mechanisms, biomarkers and targets for adult-onset Still's disease. Nat Rev Rheumatol 2018;14:603-18.

22. von Bahr Greenwood T, Palmkvist-Kaijser K, Chiang SC, et al. Elevated ferritin and soluble CD25 in critically ill patients are associated with parameters of (hyper) inflammation and lymphocyte cytotoxicity. Minerva Anestesiol 2019;85:1289-98.

23. Yao HW, Kuan CY. Early neutrophil infiltration is critical for inflammation-sensitized hypoxic-ischemic brain injury in newborns. J Cereb Blood Flow Metab 2019. [Epub ahead of print].

24. Shaaban HA, Safwat N. Mean platelet volume in preterm: a predictor of early onset neonatal sepsis. J Matern Fetal Neonatal Med 2020;33:206-11.

25. Mitrovic S, Fautrel B. New Markers for Adult-Onset Still's Disease. Joint Bone Spine 2018;85:285-93. 
Supplementary

Table S1 Clinical data of patients for FUO in subgroups

\begin{tabular}{|c|c|c|c|}
\hline Variables & Patients with infectious ( $\mathrm{N}=38$ ) & Patients with malignant $(\mathrm{N}=29)$ & Patients with connective tissue disease $(\mathrm{N}=22)$ \\
\hline Gender, female (\%) & $24(63.2)$ & $16(55.2)$ & $16(72.7)$ \\
\hline CRP & $54.93 \pm 54.85$ & $66.86 \pm 74.02$ & $97.26 \pm 69.12$ \\
\hline Ferritin $(\mu \mathrm{g} / \mathrm{L})$ & $559.87 \pm 757.02$ & $969.41 \pm 1,571.74$ & $796.24 \pm 1,206.49$ \\
\hline Lymphocyte (×10 $/ \mathrm{L})$ & $1.46 \pm 1.94$ & $1.74 \pm 2.37$ & $1.01 \pm 0.60$ \\
\hline Neutrophil $\left(\times 10^{9} / \mathrm{L}\right)$ & $4.69 \pm 3.83$ & $5.32 \pm 4.13$ & $5.58 \pm 3.30$ \\
\hline Lymphocyte\% & $24.80 \pm 20.29$ & $23.25 \pm 16.62$ & $18.69 \pm 18.73$ \\
\hline Neutrophil\% & $65.68 \pm 19.94$ & $64.07 \pm 17.95$ & $72.34 \pm 17.77$ \\
\hline PCT (\%) & $0.22 \pm 0.11$ & $0.21 \pm 0.14$ & $0.25 \pm 0.21$ \\
\hline MPV (fL) & $10.42 \pm 1.09$ & $10.32 \pm 1.05$ & $10.56 \pm 0.94$ \\
\hline PDW (\%) & $11.90 \pm 2.17$ & $12.20 \pm 2.41$ & $12.53 \pm 2.19$ \\
\hline
\end{tabular}

AOSD, adult-onset Still's disease; FUO, fever of unknown origin; WBC, white blood cell; RDW, red blood cell distribution width; PLT, platelet; PCT, plateletcrit; MPV, mean platelet volume; PDW, platelet distribution width. 